\title{
Segmentation of the Herniated Intervertebral Discs
}

\author{
Bazila \\ Department of Electronics and Communication Engineering, National Institute of Technology, \\ Hazratbal, Srinagar 190006, India \\ Email: samr_hashia@yahoo.co.in, \\ Ajaz Hussain Mir \\ Department of Electronics and Communication Engineering, National Institute of Technology, \\ Hazratbal, Srinagar 190006, India, \\ Email: ahmir@ rediffmail.com
}

Received: 22 February 2018; Accepted: 20 April 2018; Published: 08 June 2018

\begin{abstract}
This paper presents two segmentation algorithms for MR spine image segmentation helping in on time diagnosis of the spine hernia and surgical intervention whenever required. One is level set segmentation and another one is watershed segmentation algorithm. Both of these methods have been widely used before (Aslan, Farag, Arnold and Xiang, 2011) (Pan, et al., 2013) (Silvia, España, Antonio, Estanislao , and David, 2015) (Erdil, Argunşah, Ünay and Çetin, 2013) (Claudia. Et al, 2007). In our approach we have used the concept of variational level set method along with a signed distance function and is compared with the watershed segmentation which we have already implemented before on a different dataset (Hashia, Mir, 2014). In order to check the efficacy of the algorithm it is again implemented in this paper on the sagittal T2weighted MR images of the spine. It can be seen that both these methods can become very much valuable to help the radiologists with the on time segmentation of the vertebral bodies as well as of the intervertebral disks with relatively much less effort. They both are later compared with the golden standard using dice and jaccard coefficients.
\end{abstract}

Index Terms - Spine hernia, annulus fibrosus, nucleus pulposus, level set segmentation, watershed segmentation, dice coefficient, jaccard coefficient.

\section{INTRODUCTION}

Human spine provides the main support to the body that helps us to stand erect, bend, twist and also helps in providing a protective shell to the spinal cord. It consists of bones, cartilage, ligaments and muscles (Schmorl, Georg, 1959). If any of the parts of the spine are distressed by strain or injury or any disease, it can cause severe pain. Back pain is considered to be the second highest health issue after common cold and is regarded as the second most common reason why patients visit doctors' clinic in USA(Alomari, Corso, Chaudhary,
2011). It is also considered as one of the major prominent chronic diseases that causes lot of disruption in peoples' lives (Alawneh, Al-dwiekat, Alsmirat, Al-Ayyoub, 2015). The human spine is made up of 33 individual bones, called as vertebrae, interlocked with each other. These vertebrae are divided into unique regions; 24 from the top are cervical, thoracic and lumbar, which are movable, the rest 9 are sacrum and coccyx which are fused as shown in the fig 1a. Every vertebra in the spine is isolated and padded by an intervertebral disc, shielding the bones from rubbing together. The intervertebral discs are comprised of an adaptable ring of collagen strands called the annulus fibrosus which is loaded with delicate gel like substance called nucleus pulposus. The intervertebral discs are actually cartilaginous in nature and are shown in the fig $1 \mathrm{~b}$ (Michopoulou et al., 2009).

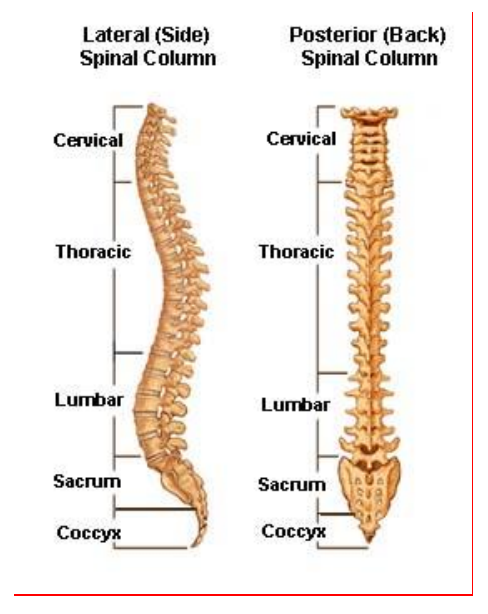

Fig.1a. Showing different parts of the spine.

Aging, trauma, genetic disorders, nutritional disorders usually result in the degeneration of the intervertebral discs (Michopoulou et al., 2009). And among all the disc degenerative disorders' disc herniation is one of the major disorders resulting in the major causes of back pain (Koh et al., 2010). In disc herniation the outer ring, i.e. the annulus fibrosus ruptures resulting in the leakage of 
the central gel-like material, the nucleus pulposus as shown in the Fig $2 \mathrm{a}$ and Fig $2 \mathrm{~b}$. In such a disorder, the gel like material puts enough pressure on the spinal nerves and causes swelling or what we call as inflammation of the nerves that are around, which results in severe back pain, radiating pain, and muscle spasm (Milette, Pierre, 2000).

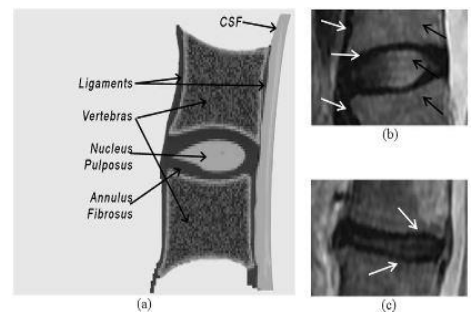

Fig.1b. Chematic of a midsagittal cut of the human spine demonstrating the major anatomical components, as well as (b) normal and (c) degenerated intervertebral disc (10. Michopoulou, et al., 2009).

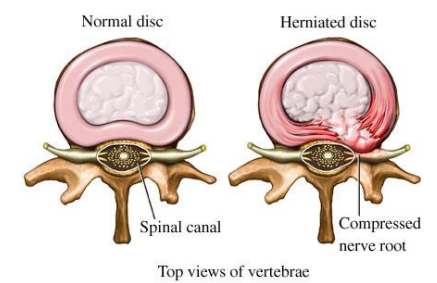

Fig.2a. Showing annulus fibrosus tear resulting in nucleus pulposus leakage and nerve compression (CBaker Chiropractic).

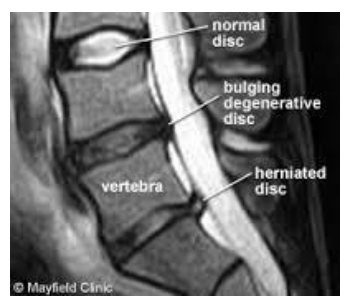

Fig.2b. MR Image showing the difference between normal and a herniated disc. There is a difference in the intensity of the signal of nucleus pulposus of normal disc and that of the nucleus pulposus of the herniated disc.

As far as diagnosis of intervertebral disc degeneration is considered MR imaging is the modality of choice (Kim et al., 1993). MR does not only help in diagnosis but an accurate segmentation of MR images of intervertebral discs can be helpful in quantification of disc degeneration and hence in a minimally invasive computer assisted spine surgery when needed (Chevrefils, Cheriet, Aubin, Grimard, 2009). Till date manual segmentation is being utilized for the measurement of the features of intervertebral discs for diagnosis and surgical purposes which is time consuming and tedious and there is a lack of reproducibility between the observers as well (Alomari, Corso, Chaudhary, Dhillon, 2014). Segmentation of MR images of spine has two major difficulties, one is: partial volume effect which ends with the blurring of intensity across the boundaries of the intervertebral discs and the other is: gray level overlapping between different tissues, for example, annulus fibrosus has gray level values similar to those of the surrounding muscles and ligaments and the gray-level values of the disc nucleus are similar to those of the vertebral bodies (Max et al., 2013). Therefore, it is difficult to indicate the exact boundary of the non-linear, posterior part of the disc, which is the most vulnerable portion of the disc for herniation. In this paper we have made an attempt to segment accurately MR images of both normal and degenerated intervertebral discs and compared the results with the manually segmented ones. Automatic segmentation is done on manually selected $\mathrm{T} 2$ weighted mid scan, sagittal MR images of the spine. Finding the exact contour of the intervertebral discs by automatic segmentation can help radiologists in making quick and accurate diagnosis of the disc herniation improving their efficacy and helping them in dealing with more number of cases. In extreme cases where there is no treatment for the herniated disc patients and surgery is recommended and automatic segmentation can be helpful in assisting surgeons in minimally invasive discectomy (Doi, Kunio, 2007). In this paper two segmentation algorithms have been implemented, one is variational level set segmentation and the other one is morphologically preprocessed watershed segmentation algorithm. Both these segmentation techniques are good in finding the contour of the objects of interest, which is the main objective in the diagnosis of the disc herniation (Carrino, John, Morrison, 2003). At the end segmentation results obtained using these algorithms are compared with the golden standard using DICE and JACCARD coefficients.

The rest of the paper is presented as: section II discusses literature review. Section III describes the methodology and its implementation. In section IV details of the clinical data set used is provided and in section $\mathrm{V}$ experimental results are given and their details are discussed in section VI and the conclusion of the work done is discussed in section VII.

\section{RELATED WORK}

A very limited work has been done on the segmentation of the herniated discs and is illustrated in the table I. The accuracy and the level of the automaticity of the disc herniation diagnosis done previously are also mentioned in the table. Tsai et al (2004) adopted a method in which geometrical features like shape, size and location were used to diagnose herniated discs 3D MRI and CT transverse sections. In another work by Koh, Jaehan, Vipin, Gurmeet (2010) diagnosis of disc herniation is done based on classifiers and features generated from spine MR images. Three different classifiers have been used: support vector machine (SVM) classifier, a perceptron classifier, and a least mean square (LMS) classifier. 68 clinical cases were studied and gave 97\% accuracy. Alomari, Corso, Chaudhary, Dhillon, (2014) used active shape model and an active contour model to help in the segmentation and feature extraction for the detection of lumbar spine disc herniation. Bayesian classifier and a Gibbs based distribution with shape potentials has also been used. Initially disc localization has been done using two level probabilistic model, earlier proposed by Corso, J. J., Raja, Chaudhary, 
Table I. Illustrating the accuracy and level of automaticity of the previous work on herniation diagnosis

\begin{tabular}{|c|c|c|c|c|c|}
\hline S.NO & PAPER & SCANS & $\begin{array}{l}\text { SCAN } \\
\text { TYPE }\end{array}$ & $\begin{array}{l}\text { ACCURA } \\
\text { CY }[\%]\end{array}$ & $\begin{array}{l}\text { AUTOMATICITY? } \\
\text { (slice, detection, segmentation) }\end{array}$ \\
\hline 1. & Tsai et al [2004] & 16 & $\begin{array}{l}\text { Axial MRI } \\
\text { or CT }\end{array}$ & $\begin{array}{l}\text { Information } \\
\text { not } \\
\text { available }\end{array}$ & Fully manual \\
\hline 2. & Koh et al [2010] & 68 & Sagittal T2 & 97 & Fully manual \\
\hline 3. & Koh et al [2012] & 70 & Sagittal T2 & 99 & Fully manual \\
\hline 4. & $\begin{array}{l}\text { Ghosh et al } \\
{[2011 \mathrm{~b}]}\end{array}$ & 35 & $\begin{array}{l}\text { Sagittal T2- } \\
\text { SPIR }\end{array}$ & 98.3 & $\begin{array}{l}\text { Mid scan, axial interaction, automatic } \\
\text { segmentation }\end{array}$ \\
\hline 5. & $\begin{array}{lll}\text { Ghosh et } & \text { al } \\
{[2011 \mathrm{a}]} & & \end{array}$ & 35 & $\begin{array}{l}\text { Sagittal T2- } \\
\text { SPIR }\end{array}$ & 94.9 & $\begin{array}{l}\text { Mid scan, axial interaction, automatic } \\
\text { segmentation }\end{array}$ \\
\hline 6. & $\begin{array}{l}\text { Alomari et al } \\
{[2010 \mathrm{a}]}\end{array}$ & 33 & $\begin{array}{l}\text { Sagittal T2- } \\
\text { SPIR }\end{array}$ & 91 & $\begin{array}{l}\text { Manual selection, information not } \\
\text { available, information not available }\end{array}$ \\
\hline 7. & $\begin{array}{l}\text { Alomari et al } \\
{[2011 \mathrm{~b}]}\end{array}$ & 65 & $\begin{array}{l}\text { Sagittal T2- } \\
\text { SPIR }\end{array}$ & 92.5 & $\begin{array}{l}\text { All slices, information not available, } \\
\text { automatic segmentation }\end{array}$ \\
\hline 8. & $\begin{array}{l}\text { Alomari et al } \\
{[2013]}\end{array}$ & 65 & $\begin{array}{l}\text { Sagittal T1- } \\
\text { T2 }\end{array}$ & 93.9 & $\begin{array}{l}\text { Manual selection, information not } \\
\text { available, automatic segmentation }\end{array}$ \\
\hline 9. & $\begin{array}{l}\text { Khaled Alawneh } \\
\text { et al [2015] }\end{array}$ & 32 & Axial MRI & 100 & Information not available \\
\hline
\end{tabular}

(2008). Alawneh, Al-dwiekat, Alsmirat, Al-Ayyoub, (2015) proposed a computer aided diagnosis system for lumbar disc hernia and have tried to extract the ROI by adaptive thresholding and determining the ROI horizontally, which means top-down MRI scans were used instead of the sagittal view, with a fixed displacement before and after the closest point to the spine. Later ROI was enhanced by CLAHE, followed by feature extraction by skeletonization. Michopoulon et al., (2009) used intensity based classifier and have implemented three different fuzzy C-means algorithms for atlas based disc segmentation. The same algorithm was also used for classifying a disc as a normal or an abnormal one.

Vaughn, M. , (2000) have done a research study on neural network for helping orthopedic surgeons in the lower back pain diagnosis and have divided lower back pain issues into 3 clinical categories. 25 features were used to train the neural network (NN) including symptoms clinical assessment results. $99 \%$ of training accuracy \& $78.5 \%$ of testing accuracy was achieved indicating training data over fitting. Bounds et al., (1990) also used $\mathrm{NN}$ for the diagnosis of disc hernia and claimed that their results were better than the golden standard but were not able to validate their system.

As per the survey the techniques implemented in this work, which are distance regularized level set segmentation and morphologically preprocessed watershed segmentation, have not been implemented for the diagnosis of the disc hernia before. Level set segmentation is the preferred segmentation algorithm as far as the contour evolution of complex topologies is considered and is able to deal with the topological changes in a better way (Qin, Zhang, 2009). There is another advantage and is that, there is no need to parameterize the points on a contour; computations are done on a fixed Cartesian grid (Xu, C., Prince, J. L., 1998). Distance regularized level set evolution has been implemented which eliminates the requirement of reinitialization resulting in reducing the computation time and induced numerical errors. As stated by (Chevrefils,
Cheriet, Aubin, Grimard, 2009) watershed segmentation technique can handle wide variety of shapes and topologies. Watershed segmentation works in poor contrast as well and hence post processing requirement is eliminated, such as joining of contours. The methodology and the implementation of both these methods are discussed in depth in the next section.

\section{METHODOLOGY AND IMPLEMENTATION}

Segmentation of medical images is a very crucial step in a multiple of medical applications (Bouzid-Daho et al., (2018). Kalaiselvi, et al., (2017). Lakshmi, et al., (2017)). There are number of automatic and semi-automatic segmentation methods, (Pan, et al., (2013), Ruiz-España, et al., (2015) Erdil, et al., (2013), Chevrefils, et al., (2007). ) and amongst them none of the methods is considered as the perfect one because of the different types of unknown noise present, poor image contrast, weal boundaries, complicated structures of the human body. Also the intensity distribution in the medical images is very complex (Balafar, et al., (2008). The methods implemented in this paper are discussed below.

\section{A. Level set segmentation}

Level set method has been initially put forward by Osher, S., Sethian, J. A., (1988). A numerical solution was proposed for processing topological changes of the contours. Level set method has been extensively used in image processing and especially in the field of image segmentation and multiple level set based segmentation algorithms have developed (Pan et al., 2013)( Ferhat et al., 2014) (Shen, Yu, Liu, Chen, 2010)( Huang, Jiang, 2009). What actually happens in level set method is that, if we have a given image $\mathrm{I}_{0}$ and $\mathrm{F}_{0}$ is its level set function which is used to interpret the aimed contour C. Each pixel of the image $I_{0}$ will have a corresponding level set function value $\left(\mathrm{F}_{0}\right)$. We define the contour as the region where level set function is zero, that implies, $\mathrm{F}_{0}=0$ and the region inside the contour is the region where level set 
function is greater than zero and the region outside the contour is less than zero. So with the change in the value of the level set function, there will be corresponding change in the region that is outside the contour and inside the contour and with result the boundary which is $\mathrm{F}_{0}=0$ will be different and hence the contour will evolve.

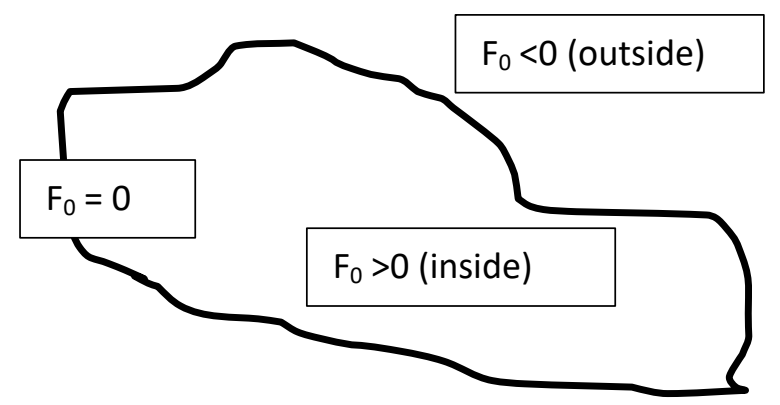

In our approach we have used the concept of the distance regularization ( $\mathrm{Li}$ et al., 2010) in which there is a concept of an external energy which drives the motion of the zero level set towards the desired location. In this approach the gradient magnitude of the level set function is forced to one of its minimum points by a term of potential function and hence maintains a desired shape of the level set function.

In practice an observed image can be expressed as

$$
\mathrm{I}=\mathrm{BI}_{\mathrm{ac}}+\mathrm{n}
$$

Where $\mathrm{I}_{\mathrm{ac}}$ is the actual image, $\mathrm{B}$ is the Bias field which can be a result of intensity inhomogeneity in MR images, $\mathrm{n}$ is the additive noise and the term $\mathrm{I}_{\mathrm{ac}}$ is an inherent physical property of the imaged objects which has $\mathrm{N}$ distinct constant values $\mathrm{C}_{1}, \ldots, \mathrm{C}_{\mathrm{N}}$ in disjoint regions $\Omega_{1}, \ldots, \Omega_{\mathrm{N}}$, that means

$$
\Omega=\cup_{i=1}^{N} \Omega_{\mathrm{i}} \quad \Omega i \cap \Omega j=\emptyset \quad i \neq j
$$

Also the shading image what we call as the bias field is varying very slowly which means ' $\mathrm{B}$ ' can be taken as a constant. In our approach standard K-means clustering (Kanungo et al., 2002) is used to classify the local intensities as because of the intensity inhomogeneity it is very difficult to segment the overlapping regions based on the pixel intensities.

The K-means clustering can be defined in a continuous form as

$$
\mathrm{E}_{\mathrm{y}}=\sum_{i=1}^{N} \int_{\Omega i=1}^{N} K(y-x)\left|I(x)-B(y) c_{i}\right|^{2} d x
$$

where $K(y-x)$ is a Gaussian function. Smaller the value of $\mathrm{E}_{\mathrm{y}}$ better the classification, therefore, it is required to minimize $\mathrm{E}_{\mathrm{y}}$ for all values of $\mathrm{y}$ in $\Omega$ and can be achieved by minimizing the integral of $\mathrm{E}_{\mathrm{y}}$ with respect to $\mathrm{y}$ and hence energy is defined as $\mathrm{E} \triangleq \int E_{y} d y$, that means,

$$
\mathrm{E}=\int\left(\sum_{i=1}^{N} \int_{\Omega i=1}^{N} K(y-x)\left|I(x)-B(y) c_{i}\right|^{2} d x\right) d y
$$

During the level set evolution $\mathrm{C}_{\mathrm{i}}$ and $\mathrm{B}$ are updated by minimizing the energy function $\mathrm{E}\left(\mathrm{F}_{0}, \mathrm{C}, \mathrm{B}\right)$

$$
\mathrm{E}\left(\mathrm{F}_{0}, \mathrm{C}, \mathrm{B}\right)=\int \sum_{i=0}^{N} e_{i}(x) M_{i}\left(F_{0}(x)\right) d x
$$

Where $\operatorname{Mi}\left(\mathrm{F}_{0}(\mathrm{x})\right)$ is the membership function which represents the phase indicator for the regions and the level set function given by $\mathrm{F}_{0}$ and $e_{i}(x)$ is defined as

$$
e_{i}(x)=\int K(y-x)\left|I(x)-B(y) c_{i}\right|^{2} d y
$$

The energy minimization with respect to the level set function $\mathrm{F}_{0}$ is given as

$$
\frac{\partial F_{0}}{\partial t}=\frac{\partial S F}{\partial t}
$$

where SF represents the speed function that controls the evolving level set.

$$
\mathrm{SF}(\mathrm{F} 0, \mathrm{C}, \mathrm{B})=\mathrm{E}+\mu \delta\left(F_{0}\right) \operatorname{div}\left(\frac{\nabla F_{0}}{\left|\nabla F_{0}\right|}\right)+\gamma \delta\left(F_{0}\right)\left|\nabla F_{0}\right|
$$

where $\delta\left(F_{0}\right)$ represents the dirac function which is the derivation of Heaviside function and $\mu, \gamma$ are the regularizing constants.

As far as the implementation in concerned, it is very straightforward. Initially scale parameters are defined. For example, sigma ' $\sigma$ ', which is the standard deviation of the Gaussian function of K-means clustering. The effect of the $\sigma$, is being discussed in detail in the section VI of the paper. The other parameters like, time step, $\nabla t$ has been put equal to 0.1 , regularization parameter $\mu$ is set equal to 1.0 and $\gamma$ is usually set to $0.001 * 255^{2}$ as a default value for most of the digital images with intensity range in [0.255]. Then level set function is initialized. After initialization Gaussian kernel is defined as a $\mathrm{w}^{*} \mathrm{~W}$ mask, where $\mathrm{w}$ is the smallest odd number, $\mathrm{w} \geq 4 * \sigma+1$. Once iteration of the level set function is started, each iteration is modeled using Gaussian probability distribution. Then the zero level contour is updated. The iteration processes is terminated once the final contour is obtained by the saturation of the energy of the object/background, or the predefined iteration limit is acquired, if not then the iteration of the level set function is again started.

\section{B. Watershed segmentation :}

Another technique, that is, watershed segmentation is implemented along with some morphological preprocessing. In the preprocessing steps image normalization is done to reduce the effect of variation in the input images. Two common methods are:

1. Contrast stretching

2. Histogram equalization

Image normalization likewise endeavors to resize the input image and henceforth decreasing the handling time, 
utilizing either closest neighbor interpolation or bilinear interpolation or bicubic interpolation. In our work Contrast-limited adaptive histogram equalization (CLAHE) was performed at the initial step for the institutionalization of the considerable number of images. Image normalization is trailed by de-noising. With a specific end goal to isolate the intervertebral plates from the vertebral bodies, the contrast enhanced images are thresholded and the level contention is registered consequently. The commotion introduce in the image can disturb the shape data; in this manner the de-noising is performed. Keeping in mind the end goal to precisely reproduce the protest shape, the de-noising process needs to enhance the signal-to-noise proportion, steadfastly. Diverse wellsprings of noise can be distinguished as: Partial volume effect, intensity inhomogeneity, presence of artifacts, closeness in gray level of different soft tissues and also the electronic noise from the instrument. Image de-noising still remains a test for specialists since noise expulsion presents ancient rarities and causes obscuring of the images, additionally de-noising procedure may expel images' fine structures. To tidy up the picture, the little particles and gaps are evacuated by doing some morphological handling, for example, a disc structuring element with radius equal to 1 and $\mathrm{N}$ equal to 0 and then image is dilated, in order to get the exact contour of the disc and the vertebrae, using again disc structuring element having radius equal to 1 and $\mathrm{N}$ equal to 0 . After de-noising border pixels are found before actualizing the watershed algorithm. Watershed segmentation was at first given by (Beucher, 1992) and till date different watershed segmentation algorithms have been actualized (Nowinski et al., 2006) (Vincent, Soille, 1991) (Haris, Efstratiadis, Maglaveras, Katsaggelos, 1998) (Huang, Chen, 2004). In our approach we have executed Fernand Meyer calculation (Meyer, 2001).

The details of the data set on which these algorithms have been implemented are provided in the next section.

\section{Clinical Data Description}

The data set used for the evaluation has MR images of 69 cases, ranging from age group of 16 to 78 years. The data set has been acquired from MEDICARE, a private diagnostic center at Srinagar, India. The sagittal, T2weighted, MR images in DICOM format, provided by MEDICARE, have matrix resolution mostly of $240 * 240$ and $256 * 205$, slice thickness ranging from $1.7 \mathrm{~mm}$ to 6 $\mathrm{mm}$ and slice gap ranging from 20 to 300 percent, TR ranging from 3.3 milliseconds to 7.8 milliseconds and TE ranging from 1.27 milliseconds to 3.69 milliseconds and flip angle ranging from 8 degrees to 20 degrees. The simulation platform used is MATLAB 2010. A total of 12000 scans are compared with the golden standard, i.e., the manually segmented images, done by the doctors at the Radiology Department of a medical institute in Kashmir, India.

\section{EXPERIMENTAL RESULTS}

To analyze visually the results, 2D images corresponding to the segmentation process in thoracic and lumbar regions are shown in the fig 3 and fig 4 below, respectively. Also the effect of the $\sigma$, i.e., the standard deviation of the Gaussian kernel used in the level set segmentation is shown in the figures.

The results obtained from the Automatic segmentation methods are compared with the golden standard. The comparison is performed using the DICE and JACCARD coefficients (Ruiz-España, Díaz-Parra, Arana, Moratal, 2015) (Michopoulou, et al., 2009) (Huang, Chu, Lai, Novak, 2009) and is given in Table II.

DICE coefficient is defined as

$$
\text { DICE }=\frac{2 *|G S \cap S e g I m|}{|G S|+\mid \text { SegIm } \mid}
$$

where GS is the golden standard and SegIm is the computed segmentation volume in voxels.

JACCARD coefficient is given by

$$
\text { JACCARD }=\frac{\mid \text { GSกSegIm } \mid}{|G S|+\mid \text { SegIm }|-| \text { GSกSegIm } \mid}
$$
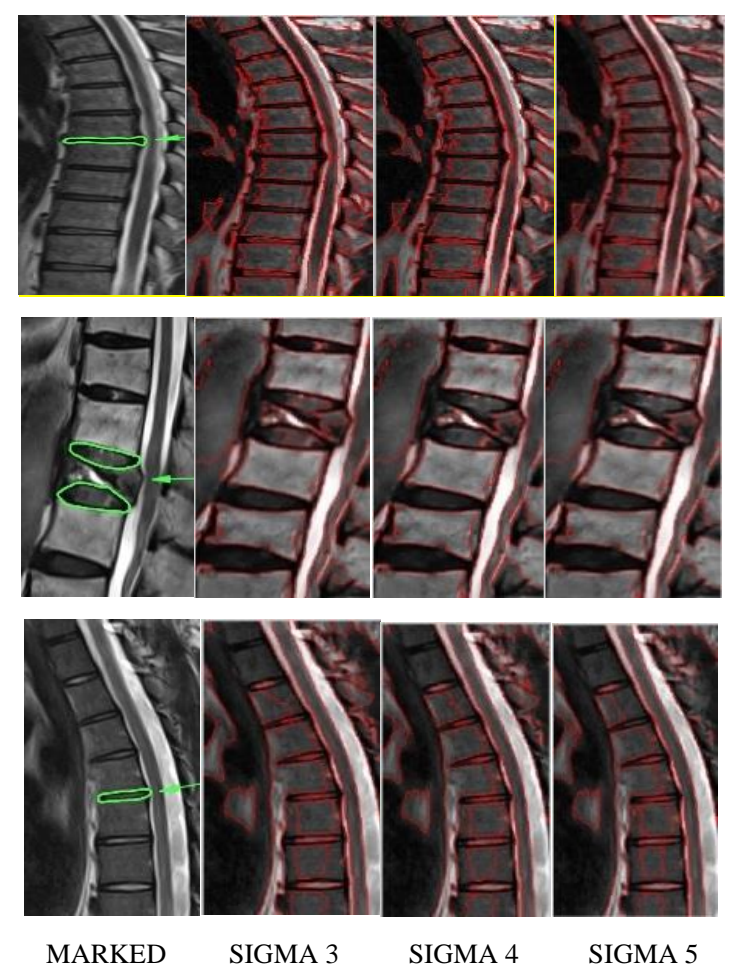

Fig.3a.Column 1: manually segmented image, column 2, 3, 4: level set segmented image of the thoracic regions at different values of $\sigma$.

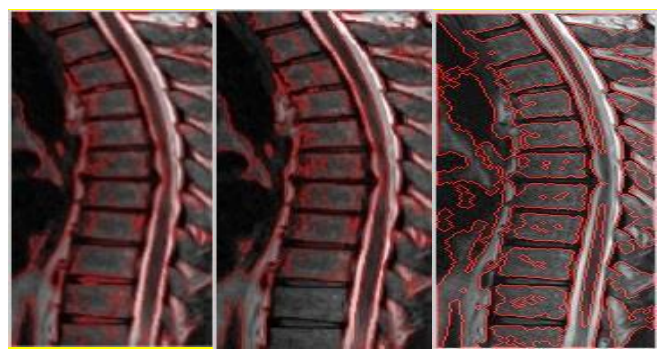



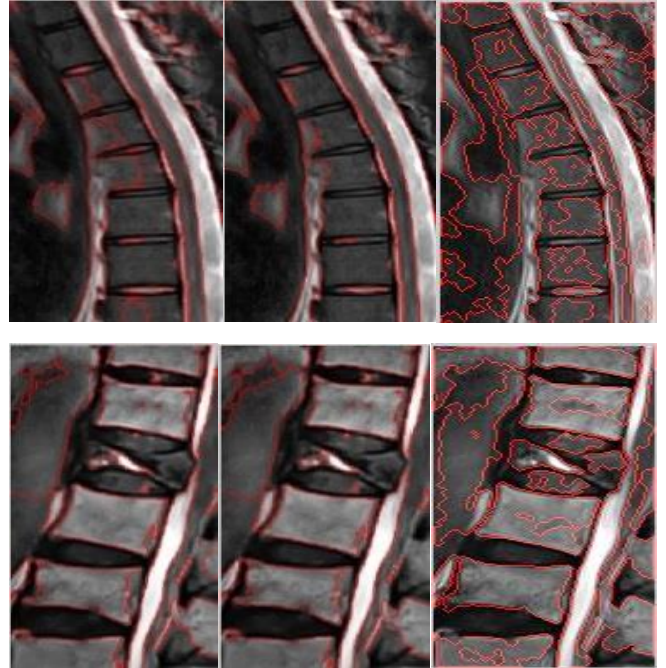

SIGMA 6

SIGMA 8

wS

Fig.3b. Column 1, 2: level set segmented image of the thoracic regions at different values of $\sigma$, column 3 : watershed segmented image.
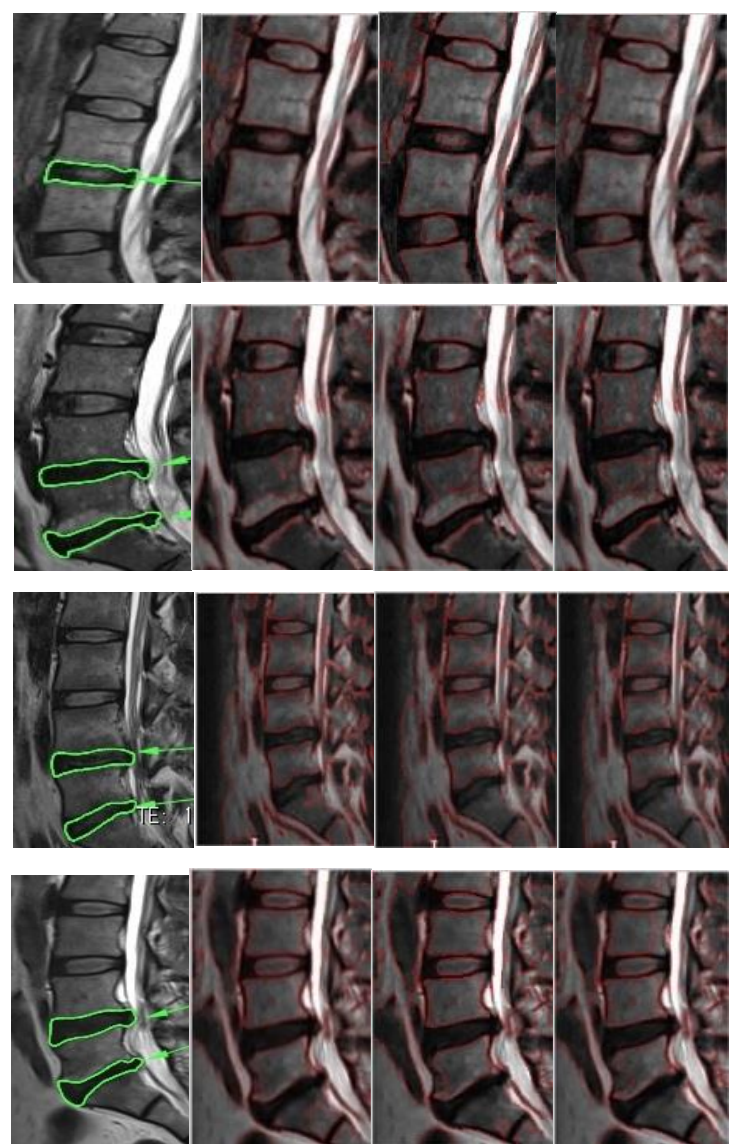

MARKED SIGMA 3 SIGMA 4 SIGMA 5

Fig.4a. Column 1: manually segmented image, column 2, 3, 4: level set segmented image of the thoracic regions at different values of $\sigma$.
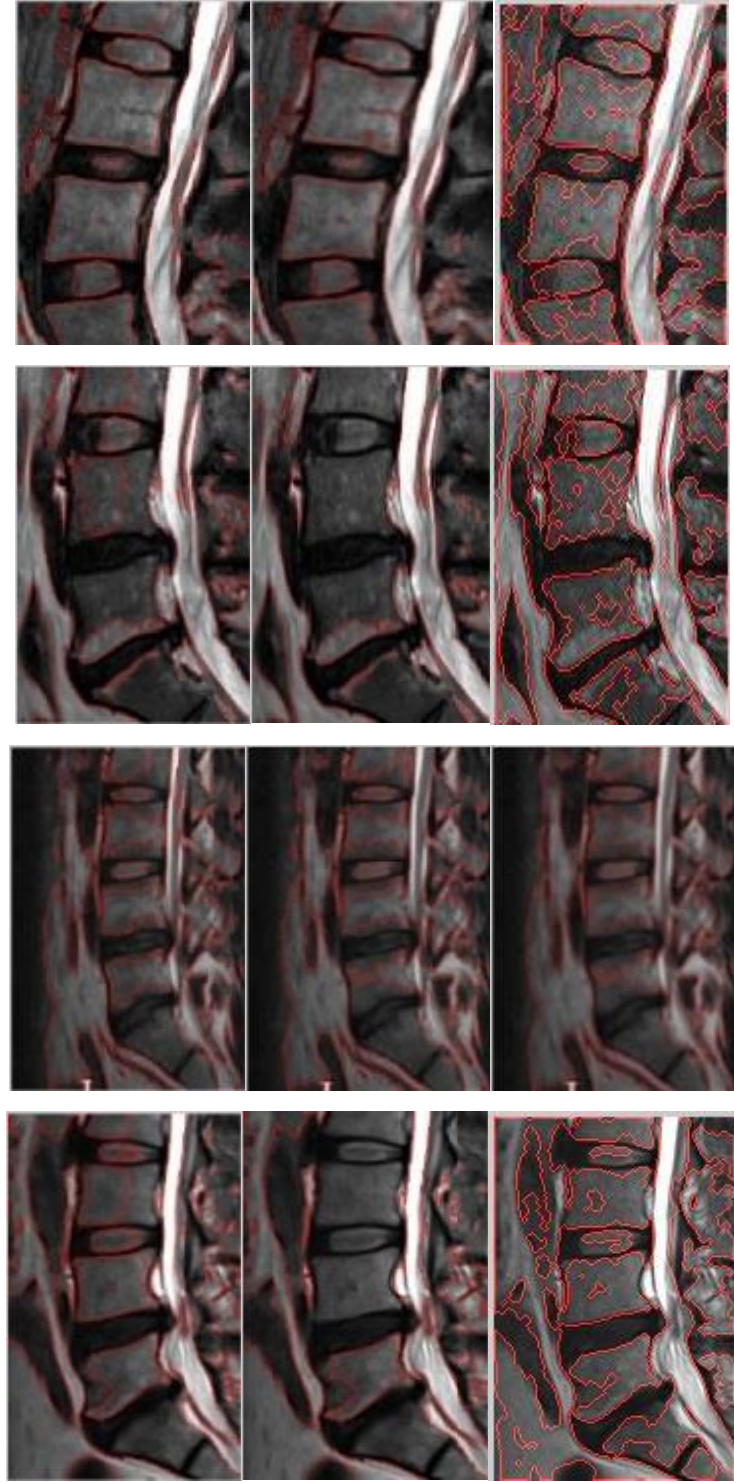

SIGMA 6

SIGMA 8

WS

Fig.4b. Column 1, 2: level set segmented image of the thoracic regions at different values of $\sigma$, column 3: watershed segmented image

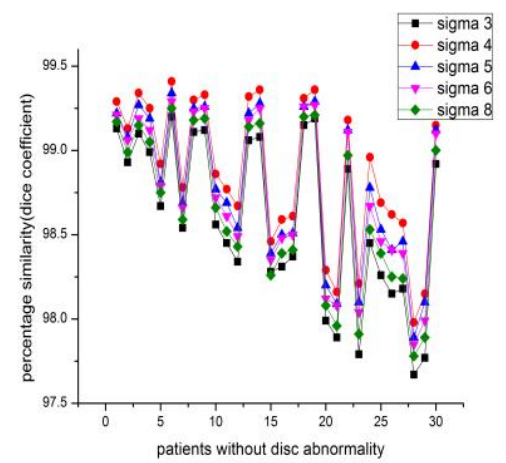




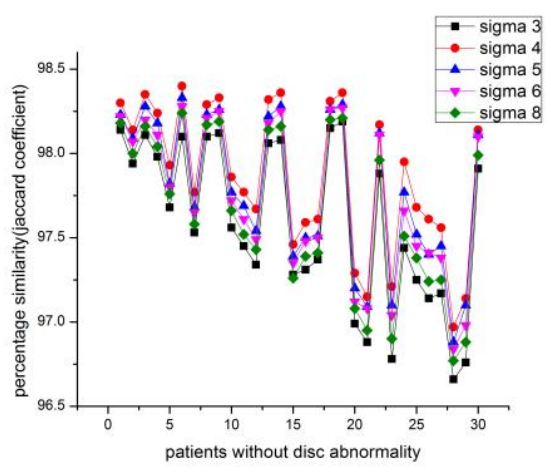

Fig.5a. Effect of sigma-patients without any abnormality.
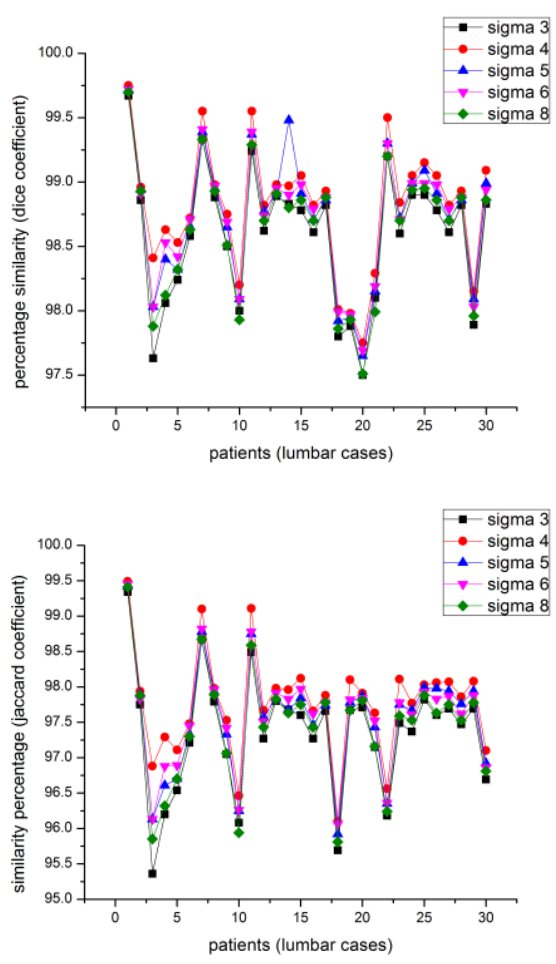

Fig.5b. Effect of sigma-patients having disc hernia in lumbar region.

These experimental results are discussed in the next section.

\section{DISCUSSION}

In sagittal, T2 weighted MR images a normal intervertebral disc appears as a bright spot at the center representing nucleus pulposus (which has $85 \%$ - 90\% water content under normal conditions) surrounded by a dark ring which represents annulus fibrosus. In disc herniation because of the disc degeneration there is a chemical change (reduced water content) in the composition of the nucleus pulposus resulting in a localized displacement of the disc material. As far as the visual inspection is considered we can observe in the fig
3 and fig 4 that the bright spot is missing in the degenerated discs, that is the nucleus pulposus is missing and the darker ring, which is the annulus fibrosus, is ruptured and is protruded outwards, stressing the spinal canal resulting in the pain and discomfort. In some cases whole nucleus pulposus is not missing, it is either deformed or a portion is missing indicating initiation of the disc hernia. In level set segmentation the results are better at $\sigma=4$, i.e., when the standard deviation of the Gaussian function of the $\mathrm{K}$ means clustering is equal to 4 , the moment we increase the value of the $\sigma$ the results get deteriorated. As can be seen from the figures 5a, 5b and $5 \mathrm{c}$, the highest peaks corresponds to $\sigma=4$. Fig 5 illustrates the percentage similarity when the segmented images are compared with the golden standard at different values of $\sigma$. Fig 5a, 5b and 5c illustrates percentage similarity of the patients without any abnormality, with abnormality in the lumbar region and the last one with abnormality in the thoracic region, respectively. . From the table II it is evident that both DICE and JACCARD coefficients support the comparison done on the visual inspection basis. It can be noted from the table II that both the similarity indices have lesser values for the thoracic region of the spine as compared to that of the lumbar region irrespective of which algorithm we have used. It can be because of the closeness of the spine in the thoracic region to the corresponding ribs (Ruiz-España, Díaz-Parra, Arana, Moratal, 2015). Another observation is that the jaccard similarity index has lesser values than corresponding dice similarity index. Jaccard index is calculated by the ratio of the intersection of the two images by their union while as the dice index is calculated by the ratio of the area of mutual overlap to the sum of the areas of the golden standard and the segmented image. Third observation is that watershed segmentation is giving better dice and jaccard coefficients as compared to the level set segmentation technique. Level set segmentation technique requires lot of fine tuning of parameters in order to obtain the desired results and also is dependent on the initial conditions while as watershed segmentation used is a morphologically preprocessed algorithm that works on the prior knowledge of the shape of the object. It can be easily noted that our techniques provide significantly improved segmentation accuracy in comparison with the disc herniation diagnosis done previously by the different authors as illustrated in the table I.

Both these algorithms are fast, unsupervised, and do produce closed contours. Level set segmentation algorithm implemented is relatively slower as compared to the watershed segmentation algorithm (Table III) but watershed segmentation has an inherent disadvantage of over-segmentation which can hinder the results.

In table III corresponding manual and automatic segmentation times are given. 
Table II. Illustration of the automatic segmentation performances.

\begin{tabular}{|c|c|c|c|c|}
\hline \multicolumn{5}{|c|}{ LEVEL SET SEGMENTATION $\sigma=3$} \\
\hline \multirow{3}{*}{$\begin{array}{l}\text { AVERAGE } \\
(\mathrm{MEAN} \pm \mathrm{SD})\end{array}$} & \multicolumn{2}{|c|}{ DICE COEFFICIENT } & \multicolumn{2}{|c|}{ JACCARD COEFFICIENT } \\
\hline & THORACIC [\%] & LUMBAR [\%] & THORACIC [\%] & $\begin{array}{l}\text { LUMBAR } \\
{[\%]}\end{array}$ \\
\hline & $98.513 \pm 0.541$ & $98.578 \pm 0.524$ & $96.87 \pm 0.93$ & $97.33 \pm 0.78$ \\
\hline MINIMUM & 97.69 & 97.5 & 95.47 & 95.36 \\
\hline MAXIMUM & 99.12 & 99.67 & 98.15 & 99.34 \\
\hline \multicolumn{5}{|c|}{ LEVEL SET SEGMENTATION $\sigma=4$} \\
\hline \multirow{3}{*}{$\begin{array}{l}\text { AVERAGE } \\
(\text { MEAN } \pm \text { SD) }\end{array}$} & \multicolumn{2}{|c|}{ DICE COEFFICIENT } & \multicolumn{2}{|c|}{ JACCARD COEFFICIENT } \\
\hline & THORACIC [\%] & LUMBAR [\%] & THORACIC [\%] & LUMBAR [\%] \\
\hline & $98.98 \pm 0.433$ & $98.80 \pm 0.4832$ & $97.5644 \pm 0.81$ & $97.769 \pm 0.73$ \\
\hline MINIMUM & 98.13 & 97.75 & 96.32 & 96.10 \\
\hline MAXIMUM & 99.49 & 99.75 & 98.72 & 99.49 \\
\hline \multicolumn{5}{|c|}{ LEVEL SET SEGMENTATION $\sigma=5$} \\
\hline \multirow{3}{*}{$\begin{array}{l}\text { AVERAGE } \\
(\text { MEAN } \pm \text { SD) }\end{array}$} & \multicolumn{2}{|c|}{ DICE COEFFICIENT } & \multicolumn{2}{|c|}{ JACCARD COEFFICIENT } \\
\hline & THORACIC [\%] & LUMBAR [\%] & THORACIC [\%] & $\begin{array}{l}\text { LUMBAR } \\
{[\%]}\end{array}$ \\
\hline & $98.72 \pm 0.50$ & $98.71 \pm 0.51$ & $97.22 \pm 0.82$ & $97.55 \pm 0.78$ \\
\hline MINIMUM & 97.92 & 97.65 & 95.93 & 95.92 \\
\hline MAXIMUM & 99.35 & 99.71 & 98.32 & 98.78 \\
\hline \multicolumn{5}{|c|}{ LEVEL SET SEGMENTATION $\sigma=6$} \\
\hline \multirow{3}{*}{$\begin{array}{l}\text { AVERAGE } \\
(\text { MEAN } \pm \text { SD) }\end{array}$} & \multicolumn{2}{|c|}{ DICE COEFFICIENT } & \multicolumn{2}{|c|}{ JACCARD COEFFICIENT } \\
\hline & THORACIC [\%] & LUMBAR [\%] & THORACIC [\%] & LUMBAR [\%] \\
\hline & $98.76 \pm 0.4747$ & $98.71 \pm 0.48$ & $97.21 \pm 0.84$ & $97.58 \pm 0.76$ \\
\hline MINIMUM & 98.09 & 97.69 & 96.26 & 96.06 \\
\hline MAXIMUM & 99.29 & 99.72 & 98.48 & 99.45 \\
\hline \multicolumn{5}{|c|}{ LEVEL SET SEGMENTATION $\sigma=8$} \\
\hline \multirow{3}{*}{$\begin{array}{l}\text { AVERAGE } \\
(\text { MEAN } \pm \text { SD) }\end{array}$} & \multicolumn{2}{|c|}{ DICE COEFFICIENT } & \multicolumn{2}{|c|}{ JACCARD COEFFICIENT } \\
\hline & THORACIC [\%] & LUMBAR [\%] & THORACIC [\%] & $\begin{array}{l}\text { LUMBAR } \\
{[\%]}\end{array}$ \\
\hline & $98.61 \pm 0.50$ & $98.62 \pm 0.51$ & $96.99 \pm 0.88$ & $97.42 \pm 0.81$ \\
\hline MINIMUM & 97.83 & 97.51 & 95.76 & 95.81 \\
\hline MAXIMUM & 99.21 & 99.70 & 98.24 & 99.41 \\
\hline \multicolumn{5}{|c|}{ WATERSHED SEGMENTATION } \\
\hline \multirow{3}{*}{$\begin{array}{l}\text { AVERAGE } \\
(\text { MEAN } \pm \text { SD) }\end{array}$} & \multicolumn{2}{|c|}{ DICE COEFFICIENT } & \multicolumn{2}{|c|}{ JACCARD COEFFICIENT } \\
\hline & THORACIC [\%] & LUMBAR [\%] & THORACIC [\%] & LUMBAR [\%] \\
\hline & $99.22 \pm 0.226$ & $99.29 \pm 0.31$ & $98.45 \pm 0.45$ & $98.6 \pm 0.61$ \\
\hline MINIMUM & 98.86 & 98.67 & 97.74 & 97.37 \\
\hline MAXIMUM & 99.44 & 99.84 & 98.89 & 99.68 \\
\hline
\end{tabular}

Table III. Manual and Automatic segmentation times

\begin{tabular}{|l|l|l|}
\hline $\begin{array}{l}\text { SEGMENTATION } \\
\text { METHOD }\end{array}$ & $\begin{array}{l}\text { MANUAL } \\
\text { INTERACTION } \\
\text { TIME (s) }\end{array}$ & $\begin{array}{l}\text { PROCESSING } \\
\text { TIME (s) }\end{array}$ \\
\hline $\begin{array}{l}\text { Level Set } \\
\text { Segmentation }\end{array}$ & 10 & 18 \\
\hline $\begin{array}{l}\text { Watershed } \\
\text { Segmentation }\end{array}$ & 10 & 2 \\
\hline Inter-observer & 150 & \\
\hline
\end{tabular}

\section{CONCLUSION}

Disc hernia is a major spine abnormality these days which results in persistent back pain and is considered to be a major reason why patients visit radiologist clinic these days. As per the researchers and projectionists there will be severe inadequacy of the radiologists in near future as the demand of radiologists is drastically increasing day by day, and is much greater than the patients (Sreeji, Vineetha, Beevi, Nasseena, 2013). Since picture archiving and communication system (PACS) has helped a lot in the retrieval and visualization part (Erdt, Steger, Sakas, 2012), so a Computer Aided Diagnosis System (CAD), which can help in generating diagnostic results from clinical MRI, CT scans or even X-rays, would reduce the burden on radiologists and can help in on time diagnosis as well in surgical intervention whenever needed. This attention has inspired the authors of the manuscript to make an attempt that would help in developing a CAD system for the diagnosis of herniated intervertebral discs. Segmentation is considered as a very essential analysis function as for as CAD system is considered. Segmentation is all about segregation of structures of interest either from the background or from each other. In medical image processing, image segmentation is a very important analysis phase for characterization of different components, such as for 
analyzing different anatomical structures, different tissue types, or different pathological regions. Image segmentation can also be helpful in image guided surgeries, tumor radiotherapies or in evaluation of therapies. Medical images mostly contain strong noise and inhomogeneity as a result segmentation is abit difficult task. In this paper we have implemented two automatic segmentation techniques for lumbar disc herniation diagnosis, and have compared the results with the golden standard. One of the segmentation algorithms is variational level set segmentation and another one is watershed segmentation technique. Implementing both these techniques can help a radiologist in quick and accurate computer aided disc herniation diagnosis and surgical intervention, whenever needed. In level set segmentation technique effect of the change in the standard deviation of the Gaussian function of $\mathrm{K}$ means clustering is also seen. With the increase in the values of the standard deviation segmentation results are getting deteriorated. Watershed segmentation technique is giving better accuracy both in the thoracic and lumbar parts of the spine as compared to the level set segmentation but has a disadvantage of over-segmentation. Future work will focus on the classification of the herniated disc and the possibility of using other modalities for diagnosis of herniated discs.

This work is an extended version of our previous work (Mir, A. H, 2014).

\section{ACKNOWLEDGMENT}

The authors wish to thank the Department of Radiology of SKIMS, Kashmir, especially Dr. Azhar for helping in evaluation.

\section{REFERENCES}

[1] Aslan, M. S., Farag, A. A., Arnold, B., \& Xiang, P. (2011, March). Segmentation of vertebrae using level sets with expectation maximization algorithm. In Biomedical Imaging: From Nano to Macro, 2011 IEEE International Symposium on (pp. 2010-2013). IEEE.

[2] Pan, Y., Feng, K., Yang, D., Feng, Y., \& Wang, Y. (2013, May). A medical image segmentation based on global variational level set. In Complex Medical Engineering (CME), 2013 ICME International Conference on (pp. 429-432). IEEE.

[3] Ruiz-España, S., Díaz-Parra, A., Arana, E., \& Moratal, D. (2015, August). A fully automated level-set based segmentation method of thoracic and lumbar vertebral bodies in Computed Tomography images. In Engineering in Medicine and Biology Society (EMBC), 2015 37th Annual International Conference of the IEEE (pp. 30493052). IEEE.

[4] Erdil, E., Argunşah, A. Ö., Ünay, D., \& Çetin, M. (2013, April). A watershed and active contours based method for dendritic spine segmentation in 2-photon microscopy images. In Signal Processing and Communications Applications Conference (SIU), 201321 st (pp. 1-4). IEEE.

[5] Chevrefils, C., Chériet, F., Grimard, G., \& Aubin, C. E. (2007, August). Watershed segmentation of intervertebral disk and spinal canal from MRI images. In International Conference Image Analysis and Recognition (pp. 10171027). Springer, Berlin, Heidelberg.
[6] Hashia, B., \& Mir, A. H. (2014, April). Segmentation of living cells: A comparative study. In Communications and Signal Processing (ICCSP), 2014 International Conference on (pp. 1524-1528). IEEE.

[7] Schmorl, G. (1959). The human spine in health and disease. New York, Grune \& Stratton.

[8] Raja'S, A., Corso, J. J., \& Chaudhary, V. (2011). Labeling of lumbar discs using both pixel-and object-level features with a two-level probabilistic model. IEEE transactions on medical imaging, 30(1), 1-10.

[9] Alawneh, K., Al-dwiekat, M., Alsmirat, M., \& AlAyyoub, M. (2015, April). Computer-aided diagnosis of lumbar disc herniation. In Information and Communication Systems (ICICS), 2015 6th International Conference on (pp. 286-291). IEEE.

[10] Michopoulou, S. K., Costaridou, L., Panagiotopoulos, E., Speller, R., Panayiotakis, G., \& Todd-Pokropek, A. (2009). Atlas-based segmentation of degenerated lumbar intervertebral discs from MR images of the spine. IEEE transactions on Biomedical Engineering, 56(9), 22252231.

[11] Koh, J., Chaudhary, V., \& Dhillon, G. (2010, March). Diagnosis of disc herniation based on classifiers and features generated from spine MR images. In Proc. of SPIE Vol (Vol. 7624, pp. 762430-1).

[12] Milette, P. C. (2000). Classification, diagnostic imaging, and imaging characterization of a lumbar herniated disk. Radiologic Clinics, 38(6), 1267-1292.

[13] Kim, K. Y., Kim, Y. T., Lee, C. S., Kang, J. S., \& Kim, Y. J. (1993). Magnetic resonance imaging in the evaluation of the lumbar herniated intervertebral disc. International orthopaedics, 17(4), 241-244.

[14] Chevrefils, C., Cheriet, F., Aubin, C. É., \& Grimard, G. (2009). Texture analysis for automatic segmentation of intervertebral disks of scoliotic spines from MR images. IEEE transactions on information technology in biomedicine, 13(4), 608-620.

[15] Alomari, R. S., Corso, J. J., Chaudhary, V., \& Dhillon, G. (2014). Lumbar spine disc herniation diagnosis with a joint shape model. In Computational Methods and Clinical Applications for Spine Imaging (pp. 87-98). Springer, Cham.

[16] Law, M. W., Tay, K., Leung, A., Garvin, G. J., \& Li, S. (2013). Intervertebral disc segmentation in MR images using anisotropic oriented flux. Medical image analysis, 17(1), 43-61.

[17] Doi, K. (2007). Computer-aided diagnosis in medical imaging: historical review, current status and future potential. Computerized medical imaging and graphics, 31(4), 198-211.

[18] Carrino, J. A., \& Morrison, W. B. (2003, December). Imaging of lumbar degenerative disc disease. In Seminars in Spine Surgery (Vol. 15, No. 4, pp. 361-383). Elsevier.

[19] Corso, J. J., Raja'S, A., \& Chaudhary, V. (2008, September). Lumbar disc localization and labeling with a probabilistic model on both pixel and object features. In International Conference on Medical Image Computing and Computer-Assisted Intervention (pp. 202-210). Springer, Berlin, Heidelberg.

[20] Tsai, M. D., Yeh, Y. D., Hsieh, M. S., \& Tsai, C. H. (2004). Automatic spinal disease diagnoses assisted by 3D unaligned transverse CT slices. Computerized Medical Imaging and Graphics, 28(6), 307-319.

[21] Vaughn, M. (2000). Using an artificial neural network to assist orthopaedic surgeons in the diagnosis of low back pain. Department of Informatics, Cranfield University (RMCS). 
[22] Bounds, D. G., Lloyd, P. J., \& Mathew, B. G. (1990). A comparison of neural network and other pattern recognition approaches to the diagnosis of low back disorders. Neural Networks, 3(5), 583-591.

[23] Qin, X., \& Zhang, S. (2009, April). New medical image sequences segmentation based on level set method. In Image Analysis and Signal Processing, 2009. IASP 2009. International Conference on (pp. 22-27). IEEE.

[24] Xu, C., \& Prince, J. L. (1998). Snakes, shapes, and gradient vector flow. IEEE Transactions on image processing, 7(3), 359-369.

[25] Li, C., Xu, C., Gui, C., \& Fox, M. D. (2010). Distance regularized level set evolution and its application to image segmentation. IEEE transactions on image processing, 19(12), 3243-3254.

[26] Ng, H. P., Ong, S. H., Foong, K. W. C., \& Nowinski, W. L. (2005). An improved watershed algorithm for medical image segmentation. In Proceedings 12th International Conference on Biomedical Engineering.

[27] Osher, S., \& Sethian, J. A. (1988). Fronts propagating with curvature-dependent speed: algorithms based on Hamilton-Jacobi formulations. Journal of computational physics, 79(1), 12-49.

[28] Pan, Y., Feng, K., Yang, D., Feng, Y., \& Wang, Y. (2013, May). A medical image segmentation based on global variational level set. In Complex Medical Engineering (CME), 2013 ICME International Conference on (pp. 429-432). IEEE.

[29] Alim-Ferhat, F., Boudjelal, A., Seddiki, S., Hachemi, B., \& Oudjemia, S. (2014, September). Wavelet Energy Embedded into a Level Set Method for Medical Images Segmentation in the Presence of Highly Similar Regions. In Mathematics and Computers in Sciences and in Industry (MCSI), 2014 International Conference on (pp. 149-153). IEEE.

[30] Shen, C., Yu, H., Liu, X., \& Chen, W. (2010, June). Medical image segmentation based on level set with new local fitting energy. In Medical Image Analysis and Clinical Applications (MIACA), 2010 International Conference on (pp. 34-37). IEEE.

[31] Huang, H., \& Jiang, J. (2009, October). Laplacian operator based level set segmentation algorithm for medical images. In Image and Signal Processing, 2009. CISP'09. 2nd International Congress on (pp. 1-5). IEEE.

[32] Li, C., Xu, C., Gui, C., \& Fox, M. D. (2010). Distance regularized level set evolution and its application to image segmentation. IEEE transactions on image processing, 19(12), 3243-3254.

[33] Kanungo, T., Mount, D. M., Netanyahu, N. S., Piatko, C. D., Silverman, R., \& Wu, A. Y. (2002). An efficient kmeans clustering algorithm: Analysis and implementation. IEEE transactions on pattern analysis and machine intelligence, 24(7), 881-892.

[34] Beucher, S. (1992). The watershed transformation applied to image segmentation. SCANNING MICROSCOPYSUPPLEMENT-, 299-299.

[35] Ng, H. P., Ong, S. H., Foong, K. W. C., Goh, P. S., \& Nowinski, W. L. (2006, March). Medical image segmentation using k-means clustering and improved watershed algorithm. In Image Analysis and Interpretation, 2006 IEEE Southwest Symposium on (pp. 61-65). IEEE.

[36] Vincent, L., \& Soille, P. (1991). Watersheds in digital spaces: an efficient algorithm based on immersion simulations. IEEE Transactions on Pattern Analysis \& Machine Intelligence, (6), 583-598.
[37] Haris, K., Efstratiadis, S. N., Maglaveras, N., \& Katsaggelos, A. K. (1998). Hybrid image segmentation using watersheds and fast region merging. IEEE Transactions on image processing, 7(12), 1684-1699.

[38] Huang, Y. L., \& Chen, D. R. (2004). Watershed segmentation for breast tumor in 2-D sonography. Ultrasound in medicine \& biology, 30(5), 625-632.

[39] Meyer, F. (2001). An overview of morphological segmentation. International journal of pattern recognition and artificial intelligence, 15(07), 1089-1118.

[40] Huang, S. H., Chu, Y. H., Lai, S. H., \& Novak, C. L. (2009). Learning-based vertebra detection and iterative normalized-cut segmentation for spinal MRI. IEEE transactions on medical imaging, 28(10), 1595-1605.

[41] Qin, X., \& Zhang, S. (2009, April). New medical image sequences segmentation based on level set method. In Image Analysis and Signal Processing, 2009. IASP 2009. International Conference on (pp. 22-27). IEEE.

[42] Mir, A. H. (2014, November). Segmentation of lumbar intervertebral discs from spine MR images. In Computational Intelligence on Power, Energy and Controls with their impact on Humanity (CIPECH), 2014 Innovative Applications of (pp. 85-91). IEEE.

[43] Sreeji, C., Vineetha, G. R., Beevi, A. A., \& Nasseena, N. (2013). Survey on Different Methods of Image Segmentation. International Journal of Scientific \& Engineering Research, 4(4).

[44] Erdt, M., Steger, S., \& Sakas, G. (2012). Regmentation: A new view of image segmentation and registration. Journal of Radiation Oncology Informatics, 4(1), 1-23.

[45] Alyahya, A. Q. (2017). Accuracy Evaluation of Brain Tumor Detection using Entropy-based Image Thresholding (Doctoral dissertation, Middle East University).

[46] BOUZID-DAHO, A., \& BOUGHAZI, M. (2018). Segmentation of Abnormal Blood Cells for Biomedical Diagnostic Aid. International Journal of Image, Graphics \& Signal Processing, 10(1).

[47] Kalaiselvi, T., Kalaichelvi, N., \& Sriramakrishnan, P. (2017). Automatic Brain Tissues Segmentation based on Self Initializing K-Means Clustering Technique. International Journal of Intelligent Systems and Applications, 9(11), 52.

[48] Lakshmi, G. A., \& Ravi, S. (2017). A double layered segmentation algorithm for cervical cell images based on GHFCM and ABC. International Journal of Image, Graphics and Signal Processing, 9(11), 39.

[49] Balafar, M. A., Ramli, A. R., Saripan, M. I., \& Mashohor, S. (2008, August). Medical image segmentation using fuzzy C-mean (FCM), Bayesian method and user interaction. In Wavelet Analysis and Pattern Recognition, 2008. ICWAPR'08. International Conference on (Vol. 1, pp. 68-73). IEEE.

\section{Authors' Profiles}

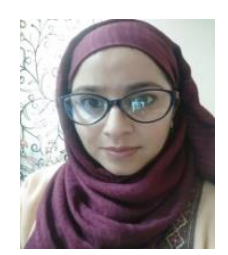

Bazila Hashia has received her $\mathrm{BE}$ in Electronics and Communication Engineering from University of Kashmir, India and then received her MTech from NIT Srinagar in the year 2010. And has worked as lecturer in the department of Electronics and Communication from Aug 2010 to Dec 2012. 
Presently she is a research scholar at NIT Srinagar in the department of Electronics and Communication. Her areas of interest are Biometrics, Image processing, patter recognition.

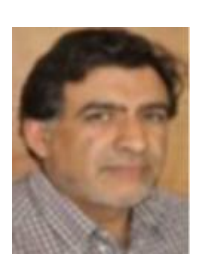

Ajaz Hussain Mir, He has done his B.E in Electrical Engineering with specialization in Electronics \& Communication Engineering (ECE). He did his M.Tech in Computer Technology and Ph.D both from IIT Delhi in the year 1989 and 1996 respectively. He is Chief Investigator of Ministry of Communication and Information Technology, Govt. of India project: Information Security Education and Awareness (ISEA). $\mathrm{He}$ has been guiding $\mathrm{PhD}$ and M.Tech thesis in Security and other related areas and has a number of International publications to his credit. Presently he is working as Professor in the Department of Electronics \& Communication Engineering at NIT Srinagar, India. His areas of interest are Biometrics, Image processing, Security, Wireless Communication and Networks.

How to cite this paper: Bazila, Ajaz Hussain Mir, " Segmentation of the Herniated Intervertebral Discs ", International Journal of Image, Graphics and Signal Processing(IJIGSP), Vol.10, No.6, pp. 31-41, 2018.DOI: 10.5815/ijigsp.2018.06.04 